\title{
Diffraction by a Half-Plane
}

\author{
By Ll. G. Chambers \\ (Received 29th March 1950. Read 5th May 1950.)
}

\section{Introduction.}

The diffraction of a simple harmonic wave train by a straightedged semi-infinite screen was originally discussed by Sommerfeld in 1895. The analysis is of a recondite character, involving the use of multivalued functions and Riemann surfaces (1). An alternative formulation of the problem is as an inhomogeneous Wiener-Hop integral equation, the solution of which also involves considerable difficulties (2). It is the purpose of this note to show that following Friedlander (3) it is possible by the use of parabolic co-ordinates to solve the problem by elementary methods. The method can be applied either to the case of sound or that of electromagnetism, the results being formally identical.

\section{Preliminary Analysis.}

We assume that the plane is defined by $x=0, y>0,-\infty<z<\infty$, the problem being two dimensional.

Let us now take parabolic co-ordinates.

$$
x=l\left(\xi^{2}-\eta^{2}\right), \quad y=2 l \xi \eta,
$$

where $l$ is any convenient quantity of the dimensions of a length.

The ranges over which $\xi, \eta$ run are $-\infty<\xi<\infty, 0 \leqq \eta<\infty$.

By restricting $\eta$ to positive values, it is possible to avoid the troubles of a two-valued function. The range of negative values of $\eta$ corresponds to the second sheet of the Riemann surface corresponding to the plane cut along the positive real axis.

We write $\quad \square_{0}^{2} g \equiv \frac{\partial^{2} g}{\partial x^{2}}+\frac{\partial^{2} g}{\partial y^{2}}-\frac{1}{c^{2}} \frac{\hat{c}^{2} g}{\partial t^{2}}$.

It can be shown that

$$
\chi=\chi(\xi, \eta)=\int_{0}^{P} F\left(c t+\overline{\xi^{2}-\eta^{2}} \cos a+2 l \xi \eta \sin a-l \zeta^{2}\right) d \zeta,
$$

where

$$
P=\left\{\xi^{2}+\gamma^{2}+\overline{\xi^{2}-\eta^{2}} \cos \alpha+2 l \xi \eta \sin \alpha\right\}^{!}
$$


is a solution of $\square_{0}^{2} \chi=0$, as is $\chi(\xi,-\eta)$ also. This may be done by direct differentiation or by using the transformation

$$
x^{\prime}=-x \sin a+y \cos \alpha, \quad y^{\prime}=x \cos a+y \sin \alpha,
$$

when $\chi$ takes the form

$$
\chi=\int_{0}^{P^{\prime}} F\left(c t+2 l \xi^{\prime} \eta^{\prime}-l \zeta^{2}\right) d \zeta
$$

where $P^{\prime}=\xi^{\prime}+\eta^{\prime}$, and then differentiating (3), $\square{ }_{0}^{2}$ being invariant with respect to the rotation of axes in the $x y$ plane.

\section{Boundary Conditions.}

Under the transformation used above, the half-plane $y=0, x>0$ goes into $\eta=0$.

Let $\Psi$ be the quantity that is under consideration. For an -.ectromagnetic case we have

$$
\Psi=E_{z} \quad \text { or } \quad \Psi=H_{z} .
$$

In the first case the boundary condition on the half-plane is $\partial \Psi / \partial y=0$, and in the second case $\Psi=0$, the half-plane being a perfect conductor in both cases. If we have a sound field and $\Psi$ is the excess pressure, then $\partial \Psi / \partial y=0$ corresponds to the case of a perfectly rigid body occupying the half-plane and $\Psi=0$ would correspond to a free surface in the half-plane (were it realisable).

The result of applying the transformation is to make the boundary $\eta=0$ and the boundary conditions

$$
\frac{\partial \Psi}{\partial \eta}=0 \quad \text { or } \quad \Psi=0 \quad \text { respectively. }
$$

The other boundary condition that applies is that when $x \rightarrow-\infty$, i.e. $\eta \rightarrow \infty$, then $\Psi \sim \Psi_{0}$, where $\Psi_{0}$ is the incident disturbance.

-4. Satisfaction of the Boundary Conditions.

For simplicity, we will only consider the case where $\Psi=0$ on $\eta=0$.

The incident field is of some arbitrary form $\Psi_{0}$ travelling in the direction $a+\pi$.

Let us consider the function

$$
\begin{aligned}
\Psi & =\frac{1}{2} \Psi_{0}\left(c t+l \overline{\xi^{2}-\eta^{2}} \cos a+2 l \xi \eta \sin a\right)-\frac{1}{2} \Psi_{0}\left(c t+l \overline{\xi^{2}-\eta^{2}} \cos a\right. \\
& +\int_{0}^{P} F\left(c t+l \overline{\xi^{2}-\eta^{2}} \cos a+2 l \xi \eta \sin a-l \zeta^{2}\right) d \zeta \\
& -\int_{0}^{Q} F\left(c t+l \overline{\xi^{2}-\eta^{2}} \cos \alpha-2 l \xi \eta \sin a-l \zeta^{2}\right) d \zeta,
\end{aligned}
$$




$$
\begin{aligned}
& P=\left\{\xi^{2}+\eta^{2}+\overline{\xi^{2}-\eta^{2}} \cos \alpha+2 \xi \eta \sin \alpha\right\}^{1} \rightarrow \infty \text { as } \eta \rightarrow \infty, \\
& Q=\left\{\xi^{2}+\eta^{2}+\overline{\xi^{2}-\eta^{2}} \cos a-2 \xi \eta \sin \alpha\right\}^{1} \rightarrow-\infty \text { as } \eta \rightarrow \infty .
\end{aligned}
$$

It is obvious by inspection that

$$
\Psi \text { satisfies } \square_{0}^{2} \Psi=0, \quad \text { with } \Psi=0 \text { on } \eta=0 .
$$

In order to satisfy the condition $\Psi \sim \Psi_{0}$ as $\eta \rightarrow \infty$ we must have $\Psi_{0}\left(c t+l \overline{\xi^{2}-\eta^{2}} \cos \alpha+2 l \xi \eta \sin \alpha\right)$

$=\frac{1}{2} \Psi_{0}\left(c t+l \overline{\xi^{2}}-\eta^{2} \cos \alpha+2 l \xi \eta \sin \alpha\right)-\frac{1}{2} \Psi_{0}\left(c t+l \overline{\xi^{2}-\eta^{2}} \cos \alpha\right.$

$+\int_{0}^{\infty} F\left(c t+l \overline{\xi^{2}-\eta^{2}} \cos \alpha+2 l \xi \eta \sin \alpha-l \zeta^{2}\right) d \zeta$

$-\int_{0}^{\infty} F\left(c t+l \overline{\xi^{2}-\eta^{2}} \cos a-2 l \xi \eta \sin \alpha-l \zeta^{2}\right) d \zeta$

for $\eta \rightarrow \infty$.

The functions $\Psi_{0}$ and $F$ must therefore be related by

$$
\frac{1}{2} \Psi_{0}(\mu)=\int_{0}^{\infty} \dot{F}\left(\mu-l \zeta^{2}\right), d \zeta+C .
$$

As explained in Friedlander's paper (3) the arbitrary constant can be ignored, and the solution of the integral equation is given by

$$
F(u)=\frac{1}{\pi} l^{\frac{1}{2}} \int_{-\infty}^{u} \frac{\Psi_{0}^{\prime}(z) d z}{(u-z)^{\frac{1}{2}}}=\frac{2 l^{\frac{1}{2}}}{\pi} \int_{0}^{\infty} \Psi_{0}^{\prime}\left(u-t^{2}\right) d t
$$

provided that

$$
F(-\infty)=0 \text {. }
$$

It is clear by inspection of (A) that if we have a continuous wave of circular frequency $\omega=k c, \Psi_{0}=\exp (i k \mu)$, then the solution is

$$
F(u)=\sqrt{2} \exp (i \pi / 4) \exp (i k \mu),
$$

which in fact satisfies (B) even though (C) is not true.

If we take $\Psi_{0}(\mu)=\exp \left(i k_{\mu}\right)$, our incident wave is

$$
\exp \left\{i \hbar\left(c t+l \overline{\xi^{2}-\eta^{2}} \cos \alpha+2 l \zeta \eta \sin \alpha\right)\right\}
$$

which is a plane wave travelling in the direction $a+\pi$,

$$
\begin{aligned}
\Psi & =\frac{1}{2} \exp \{i k \rho \cos (\phi-a)\}-\frac{1}{2} \exp \{i k \rho \cos (\phi+u)\} \\
& +\exp \{i k \rho \cos (\phi-a)\} v^{\prime} \exp (i \pi / 4) \int_{0}^{P} \exp \left(-i k l \zeta^{2}\right) d \zeta \\
& -\exp \{i k \rho \cos (\phi+a)\} \sqrt{2} \exp (i \pi / 4) \int_{0}^{Q} \exp \left(-i k l \zeta^{2}\right) d \zeta,
\end{aligned}
$$

the time factor having been dropped. 
Up till now $l$ has been an arbitrary length. It will be convenient to take it as the warelength $(k l=k \lambda=2 \pi)$,

$$
\begin{aligned}
P & =\left\{\xi^{2}-\eta^{2}+\overline{\xi^{2}-\eta^{2}} \cos \alpha+2 \xi \eta \sin \alpha\right\}^{\frac{1}{2}} \\
& =(\rho+x \cos \alpha+y \sin \alpha)^{k} / \lambda^{t} \\
& =(\rho / \lambda)^{t}[1+\cos (\phi-\alpha)]^{\ddagger}=(2 \rho / \lambda)^{\frac{1}{2}} \cos \frac{1}{2}(\phi-\alpha) .
\end{aligned}
$$

In a similar manner we find that

$$
Q=(2 \rho / \lambda)^{\frac{1}{2}} \cos \frac{1}{2}(\phi+a) \text {. }
$$

By a little manipulation we get

$$
\begin{aligned}
\Psi & =\frac{1}{2} \exp \{i k \rho \cos (\phi-a)\}-\frac{1}{2} \exp \{i k \rho \cos (\phi+a)\} \\
& +\exp \{i k \rho \cos (\phi-\sigma)\} \frac{1}{\sqrt{ } \pi} \exp (i \pi / 4) \int_{0}^{p} \exp \left(-i \gamma^{2}\right) d \gamma \\
& -\exp \{i k \rho \cos (\phi+a)\} \frac{1}{\sqrt{ } \pi} \exp (i \pi / 4) \int_{0}^{q} \exp \left(-i \gamma^{2}\right) d \gamma
\end{aligned}
$$

where $\quad p=\sqrt{2} \overline{k \rho} \cos \frac{1}{2}(\phi-a), \quad q=\sqrt{2 k \rho} \cos \frac{1}{2}(\phi+a)$.

By making use of the fact that

$$
1=\frac{2}{\sqrt{ }-(i \pi)} \int_{-\infty}^{0} \exp \left(-i \gamma^{2}\right) d \gamma
$$

we have immediately

$$
\begin{aligned}
\beta \Psi=\exp \{i k \rho \cos (\phi-\alpha)\} \int_{-\infty}^{p} & \exp \left(-i \gamma^{2}\right) d \gamma \\
& \quad-\exp \{i k \rho \cos (\phi+a)\} \int_{-\infty}^{q} \exp \left(-i \gamma^{2}\right) d \gamma
\end{aligned}
$$

where $\beta=\sqrt{\pi} \exp (-i \pi / 4)$.

It is fairly obvious that for the boundary condition $\partial \Psi / \partial \eta=0$ on $\eta=0$ we carry through an almost identical analysis beginning with the quantity

$$
\begin{aligned}
\Psi & =\frac{1}{2} \Psi_{0}\left(c t+l \overline{\xi^{2}-\eta^{2}} \cos a+2 l \xi \eta \sin \alpha\right)+\frac{1}{2} \Psi_{0}\left(c t+l \overline{\xi^{2}-\eta^{2}} \cos \alpha\right. \\
+ & \int_{0}^{r} F\left(c t+l \xi \overline{\xi^{2}-\eta^{2}} \cos \alpha+2 l \xi \eta \sin \alpha-l \zeta^{2}\right) d \zeta \\
+ & \int_{0}^{Q} F\left(c t+l \xi^{2}-\overline{\left.\eta^{2} \cos \alpha-2 l \xi \eta \sin \alpha-l \zeta^{2}\right) d \zeta .}\right. \\
& \text { We arrive at the result }
\end{aligned}
$$

$$
\begin{aligned}
\beta \Psi=\exp \{i k \rho \cos (\phi-\alpha)\} & \int_{-\infty}^{p} \exp \left(-i \gamma^{2}\right) d \gamma \\
& +\exp \{i k \rho \cos (\phi+\alpha)\} \int_{-\infty}^{q} \exp \left(-i \gamma^{2}\right) d \gamma,
\end{aligned}
$$

$P, Q, p, q, \beta$ having the same meanings as before. 
5. Expressions for other field eomponents.

The results of the foregoing analysis may be used to give formulæ for the field components which are not parallel to the edge of the halfplane. The two cases of electric vector perpendicular and parallel to the edge will be considered. In order to write down formulæ for these components, it is first necessary to write down the results of certain differentiations. Since

$$
\frac{\partial F}{\partial x}=\cos \phi \frac{\partial F}{\partial \rho}-\sin \phi \frac{1}{\rho} \frac{\partial F}{\partial \phi}, \quad \frac{\partial F}{\partial y}=\sin \phi \frac{\partial F}{\partial \rho}+\cos \phi \frac{1}{\rho} \frac{\partial F}{\partial \phi},
$$

the values of $\frac{\partial G}{\partial x}, \frac{\partial G}{\partial \bar{y}}$ can be written down fairly easily, where

and

$$
G=\exp \{i k \rho \cos (\phi+\chi)\} \int_{-\infty}^{m} \exp \left(-i \gamma^{2}\right) d \gamma
$$

These values are given by

$\frac{1}{i k} \frac{\partial G}{\partial x}=G \cos \chi+\sqrt{1 / 2 k \rho} \exp \left\{-i\left[k \rho+\frac{1}{2} \pi\right]\right\} \cos \frac{1}{2}(\phi-\chi)$

and

$-\frac{1}{i k} \frac{\partial G}{\partial y}=G \sin \chi+\sqrt{1 / 2 k \rho} \exp \left\{-i\left[k \rho-\frac{1}{2} \pi\right]\right\} \sin \frac{1}{2}(\phi-\chi)$.

We again write

$$
\beta=\sqrt{\pi} \exp (-i \pi / 4)
$$

We write also

$$
\begin{aligned}
& G_{+}=\exp \{i k \rho \cos (\phi+a)\} \int_{-\infty}^{q} \exp \left(-i \gamma^{2}\right) d \gamma \\
& G_{-}=\exp \{i k \rho \cos (\phi-a)\} \int_{-\infty}^{p} \exp \left(-i \gamma^{2}\right) d \gamma \\
& \cdot p=\sqrt{2 k \rho} \cos \frac{1}{2}(\phi-a), \quad q=\sqrt{2 k_{\rho}} \cos \frac{1}{2}(\phi+a) .
\end{aligned}
$$

(A) Electric vector perpendicular to edge.

The components which are of interest in this case are related by

$$
E_{x}=Z_{0} \frac{1}{i k} \frac{\partial H_{z}}{\partial y}, \quad E_{y}=-Z_{0} \frac{1}{i k} \frac{\partial H_{z}}{\partial x} .
$$

The incident field is defined by

$$
\begin{aligned}
Z_{0} H_{z} & =E_{0} \exp \{i k(x \cos \alpha+y \sin \alpha)\} \\
& =E_{0} \exp \left\{i k_{\rho} \cos (\phi-a)\right\}
\end{aligned}
$$




$$
\begin{aligned}
& E_{x}=E_{0} \exp \{i k \rho \cos (\phi-a)\} \sin \alpha \\
& E_{y}=-E_{0} \exp \{i k \rho \cos (\phi-a)\} \cos \alpha .
\end{aligned}
$$

The diffracted field is defined by

$$
\beta Z_{0} H_{z}=E_{0}\left(G_{-}+G_{+}\right),
$$

and performing the necessary differentiations we deduce that $\beta E_{x} / E_{0}=\sin \alpha\left(G_{-}-G_{+}\right)$

$-\sqrt{2 / k_{\rho}} \sin \frac{1}{2} \phi \cos \frac{1}{2} \alpha \exp \left\{-i\left(k \rho-\frac{1}{2} \pi\right)\right\}$

$$
\begin{aligned}
\beta E_{y} / E_{0}= & -\cos \alpha\left(G_{-}+G_{+}\right) \\
& -\sqrt{2 / k \rho} \exp \left\{-i\left(k \rho+\frac{1}{2} \pi\right)\right\} \cos \frac{1}{2} \phi \cos \frac{1}{2} \alpha .
\end{aligned}
$$

(B) Electric vector parallel to edge.

The components which are of interest in this case are related by

$$
H_{x}=-Z_{0}^{-1} \frac{1}{i k} \frac{\partial E_{z}}{\partial y}, \quad H_{y}=Z_{0}^{-1} \frac{1}{i k} \frac{\partial E_{z}}{\partial x} .
$$

The incident field is defined by

$$
\begin{aligned}
E_{z} & =E_{0} \exp \{i k(x \cos \alpha+y \sin \alpha)\} \\
& =E_{0} \exp \{i k \rho \cos (\phi-\alpha)\} \\
Z_{0} H_{x} & =-E_{0} \exp \{i k \rho \cos (\phi-a)\} \sin \alpha \\
Z_{0} H_{y} & =E_{0} \exp \{i k \rho \cos (\phi-a)\} \cos \alpha .
\end{aligned}
$$

The diffracted field is defined by

$$
\beta E_{z}=E_{0}\left(G_{-}-G_{+}\right),
$$

and performing the necessary differentiations we deduce that $\beta Z_{0} H_{x} / E_{0}=\sin \alpha\left(G_{-}+G_{+}\right)$

$$
+\sqrt{ } 2 / \overline{k \rho} \exp \left\{-i\left(k \rho-\frac{1}{2} \pi\right)\right\} \cos \frac{1}{2} \phi \sin \frac{1}{2} \alpha
$$

$\beta Z_{0} H_{y} / E_{0}=\cos \alpha\left(G_{-}-G_{+}\right)$

$$
-\sqrt{2 / k \rho} \exp \left\{-i\left(k \rho+\frac{1}{2} \pi\right)\right\} \sin \frac{1}{2} \phi \sin \frac{1}{2} \alpha \text {. }
$$

(C) Fresnel approximation.

The Fresnel approximation, being scalar, is applicable to any of the six components of the electromagnetic field.

If $\Psi_{0}=\exp \{i k(x \cos a+y \sin a)\}$ defines any incident field 
component in the absence of a screen, then, to the first order, the field in $y<0$ is given by

$$
\beta \Psi=\Psi_{0} \int_{-\infty}^{s} \exp \left(-i \gamma^{2}\right) d \gamma
$$

where $S=\sqrt{2 \hbar \frac{1}{2}} \chi$ and $\chi=\pi-(\phi-a), \rho$ being assumed large and $\chi$ being assumed small.

Now if $x$ is small

$$
\frac{1}{2} \chi \sim \sin \frac{1}{2} \chi=\cos \frac{1}{2}(\pi-\chi)=\cos \frac{1}{2}(\phi-a)
$$

and so

$$
S \sim \sqrt{2 k_{\rho}} \cos \frac{1}{2}(\phi-\alpha) .
$$

Thus the Fresnel approximation gives the approximate result that $\beta \Psi=G_{-}$.

Un examining the results obtained from the exact theory, we - Iserve that $G_{-} \beta^{-1}$ is common to all the expressions for the six field components.

\section{Discussion.}

It is clear from the form of the result that, provided the integral equation (A) is soluble, the method of solution will hold for any shape to the incident pulse, as has been indicated by Friedlander.

Furthermore, it is possible, formally at any rate, to obtain the diffracted field from any incident field; for if we have an incident field

$$
\Psi_{0}(x, y)=\int_{C} A(\alpha) \exp \{i k(x \cos \alpha+y \sin \alpha)\} d \alpha
$$

then the resultant field will be obtained by taking the diffracted field for a plane wave, multiplying by $A(\alpha)$ and integrating with respect to $a$ over the proper contour $\mathrm{C}$ in the complex $a$-plane.

The use of parabolic co-ordinates in this problem of the halfplane obviates the use of the two-valued function of Sommerfeld, and the line $\eta=0$ corresponds to the slit in the Riemann surface at which the two sheets coalesce, negative $\eta$ corresponding to the unused sheet.

Another formulation involves the replacing of the plane (for an electromagnetic field) by a sheet of magnetic or electric current and writing down the condition that, for a certain component, the sum of the incident field and the field due to the sheet vanishes along the sheet, thereby giving an integral equation of the Wiener-Hopf type for the current distribution (2). Such an integral equation is soluble only with considerable difficulty. 
Examination of the conditions $p \gtrless 0, q \gtreqless 0$ gives us further insight into the physical conditions involved. It is clear that

$$
\frac{1}{\beta} \int_{-\infty}^{T} \exp \left(-i \gamma^{2}\right) d \gamma \rightarrow \begin{array}{ll}
1 & (T \rightarrow+\infty) \\
0 & (E \rightarrow-\infty) .
\end{array}
$$

If we now consider as an example the case where $\Psi=0$ on $\eta=0$ (the other case is very similar), we have three separate cases: (1) $p>0, q>0$. At large distances from the origin the field is effectively

$$
\exp \left\{i k_{\rho} \cos (\phi-a)\right\}-\exp \left\{i k_{\rho} \cos (\phi+a)\right\}
$$

which is the same as that for reflection by a complete plane.

(2) $p>0, q<0$. At large distances from the origin the field is effectively

$$
\exp \{i k \rho \cos (\phi-a)\}
$$

which is the same as the incident field.

(3) $p<0, q<0$. This is the geometrical shadow region and at large distances from the origin the field is effectively zero, the region being screened by the half plane.

7. Acknowledgment.

This paper is published by kind permission of the Lords Commissioners of the Admiralty, the work having been done when the author was a member of the Royal Naval Scientific Service.

\section{REFERENCES.}

(1) See for example, Handbuch der Physik, Vol. XX,263-291, "Licht als Wellenbewegung."

(2) Copson, 1946. "An integral equation method of solving plane diffraction problems," Proc. Roy. Soc., A 186, 100-118.

(3) Friedlander, 1946. "The diffraction of sound pulses. I. Diffraction by a semiinfinite plane," Proc. Roy. Soc., A 186, 322-344.

Royal Military College of Science, ShrivenhaM, Wilts. 